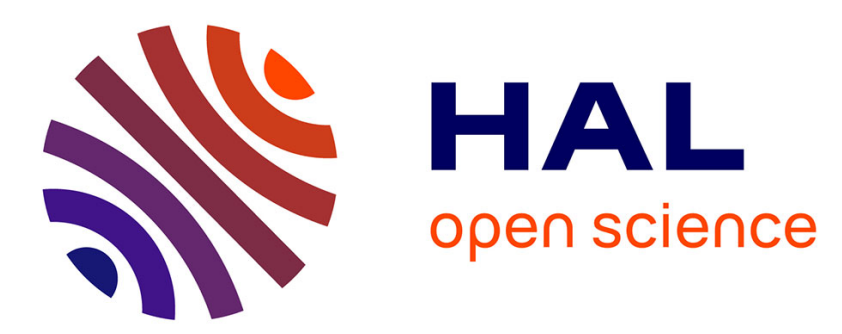

\title{
Observability forms for switched systems with Zeno phenomenon or high switching frequency
}

Lei Yu, Jean-Pierre Barbot, Driss Boutat, Djamila Benmerzouk

\section{To cite this version:}

Lei Yu, Jean-Pierre Barbot, Driss Boutat, Djamila Benmerzouk. Observability forms for switched systems with Zeno phenomenon or high switching frequency. IEEE Transactions on Automatic Control, 2011, 56 (2), pp.436 - 441. 10.1109/TAC.2010.2090066 . inria-00530112

\section{HAL Id: inria-00530112 \\ https://hal.inria.fr/inria-00530112}

Submitted on 27 Oct 2010

HAL is a multi-disciplinary open access archive for the deposit and dissemination of scientific research documents, whether they are published or not. The documents may come from teaching and research institutions in France or abroad, or from public or private research centers.
L'archive ouverte pluridisciplinaire $\mathbf{H A L}$, est destinée au dépôt et à la diffusion de documents scientifiques de niveau recherche, publiés ou non, émanant des établissements d'enseignement et de recherche français ou étrangers, des laboratoires publics ou privés. 


\title{
Observability forms for switched systems with Zeno phenomenon or high switching frequency
}

\author{
L. Yu, J.-P. Barbot ${ }^{\dagger \ddagger}$ D. Boutat\$ $\$$ D. Benmerzouk
}

October 27, 2010

\begin{abstract}
This paper deals with the observability of a class of switched systems with Zeno phenomenon or high switching frequency. Particularly, three observability forms are proposed and the observability for each form with knowledge of filtered switching signal is analyzed. Meanwhile, sufficient and necessary conditions for the existence of a diffeomorphism to transform a class of switched systems into one of such forms are presented. Examples and simulations are given at the end to highlight the theoretical results.
\end{abstract}

\section{Introduction}

Even though Zeno phenomenon is due to the high abstraction when modeling physical systems [22], it is still interesting to analyze the observability and design an observer for system with Zeno behavior, since some physical systems may have behavior close to Zeno. On the other hand, the work concerning about the observability for systems with Zeno behavior can also be adopted to analyze that of systems with high switching frequency.

Since at least fifty years ago, some particular types of Zeno phenomenon have been studied [8, 9, 11, 17]. The first order sliding mode [20] and the high order sliding mode $[7,16]$ have been largely applied in control and observer design for many years, and correspondingly, by considering solutions of differential equations in the sense of Fillipov [2], the main property of sliding mode is the

${ }^{*}$ L.Yu is with Signal Processing Laboratory, Electronic and Information School, Wuhan University, China, and ECS-Lab EA 3649, ENSEA, 6 Avenue du Ponceau, 95014 CergyPontoise, France.lei.yu@ensea.fr

${ }^{\dagger}$ J.-P. Barbot is with ECS-Lab EA 3649, ENSEA, 6 Avenue du Ponceau, 95014 CergyPontoise, France. barbot@ensea.fr

${ }^{\ddagger}$ L. Yu and J.-P. Barbot are with the EPI ALIEN, INRIA, France.

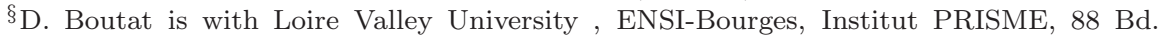
Lahitolle, 18020 Bourges Cedex, France. driss.boutat@ensi-bourges.fr

"D. Benmerzouk is with Department of Mathematics, Tlemcen University, Algeria. d_benmerzouk@yahoo.fr 
convergence of the system behavior in finite time under some controllability (or observability) matching conditions $[6,19]$.

In [1], the Zeno phenomenon is categorized to "Chattering" and "Genuinely" Zeno. Analytically, the Chattering Zeno corresponds to both of the first order sliding mode and the high order sliding mode on the sliding manifold, while Genuinely Zeno corresponds to the high order sliding mode just before reaching the sliding manifold $[16,19]$. From this point of view, this paper presents observability conditions for switched system with Zeno phenomenon through both sliding mode and normal form approaches.

For the sake of simplicity, output switches and continuous state jumps are not considered in this paper ${ }^{1}$. Let us consider the following class of switched system $S$ :

$$
\left\{\begin{array}{l}
\dot{x}=f_{q}(x) \\
y=h(x)
\end{array}\right.
$$

where $x(t) \in U \subset \mathbb{R}^{n}$ is the state, $y(t) \in \mathbb{R}$ is the measured output, vector fields $f_{q}: U \rightarrow \mathbb{R}^{n}, q \in Q=\{1, \ldots, N\}$ and function $h: U \rightarrow \mathbb{R}$ are sufficiently smooth for each $q$. The discrete state $q$ may be driven by a switching function $\sigma: \mathbb{R} \rightarrow Q$.

From geometrical view point, through a diffeomorphism $z=\phi(x)$, one can transform the switched system $S$ into some observability forms, i.e. some new system $S^{\prime}$, with following expression:

$$
\left\{\begin{array}{l}
\dot{z}=g_{q}(z) \\
y=z_{1}
\end{array}\right.
$$

where $z(t) \in V \subset \mathbb{R}^{n}$ is the new state, vector fields $g_{q}: V \rightarrow \mathbb{R}^{n}$ with $q \in Q$, and $z_{1}$ is the first new state.

In this paper, two basic observability forms are proposed [21]: the first basic observability form is directly inspired by the observer matching condition, and the second basic observability form is by differential geometric results [15, 14]. Moreover, Henstock-Kurzweil (HK) integral [12] is considered instead of the notion of Lebesgue integral, since HK integral much more general than Riemman and Lebesgue integral. Consequently, the analytical representation of the switching signal would be more suitable for special discontinuous signals, for instance the infinite switching signal. Meanwhile, the two basic forms can be easily extended to a more general form, called the "extended observability form". Specifically, we denote $S^{\prime}(1), S^{\prime}(2), S^{\prime}(3)$ the first basic, the second basic and the extended observability form.

In order to deal with the observability of system $S$, the following assumption for each subsystem is needed throughout this paper.

\section{Assumption 1}

Regularly Weakly Locally Observable for Subsystems: For each $q \in Q$ the pair

\footnotetext{
${ }^{1}$ The bouncing ball can be treated as a particular switched system with state jump at the switching time.
} 
$\left(y, f_{q}\right)$ is Regularly Weakly Locally Observable ${ }^{2}$ (RWLO), i.e. $\operatorname{rank}\left\{d L_{f}^{j} h, j=\right.$ $0, \ldots, n-1\}=n$.

This paper is organized as follows. In section 2, three observability forms are proposed and their corresponding conditions for the existence of a diffeomorphism which transforms the switched system into one of such forms are given. In section 3, the observability for the considered system is analyzed. Simulations are given in section 4 .

\section{Observability forms and conditions for exis- tence of a diffeomorphism}

In this section, three observability forms are presented respectively. Although the first two basic observability forms have already been discussed in [21], a new extended observability form with more general form is given here and moreover, the proofs for the theorems are rearranged. On the other hand, one approach to find the required vector fields in the theorems is given (see Remark 1).

\subsection{First basic observability form}

The first basic observability form is inspired by observer matching condition, and its vector field $g_{q}$ is written as follows:

$$
S^{\prime}(1): \quad g_{q}(z)=A_{n n} z+B_{n} \chi(z)
$$

where $A_{n n}$ is a $n \times n$ superior nilpotent Jordan matrix, written as

$$
\begin{gathered}
A_{n n}=\left[\begin{array}{cccc}
0 & 1 & & \\
& 0 & \ddots & \\
& & \ddots & 1 \\
& & & 0
\end{array}\right]_{n \times n} \\
B_{n}=(0,0, \ldots 1)^{T} \text { and } \chi_{q}(z) \in \mathbb{R} .
\end{gathered}
$$

Theorem 1 Under Assumption 1, there exists a diffeomorphism $z=\phi(x)$ which transforms the system $S$ into $S^{\prime}(1)$ if and only if

$$
L_{f_{\Delta_{\kappa}, \nu}} L_{f_{\kappa}}^{i-1} h=0, \quad \forall \kappa, \nu \in Q \text { and } \forall i \in\{1, \ldots n-1\}
$$

where $f_{\Delta_{\kappa, \nu}}(x)=f_{\kappa}(x)-f_{\nu}(x)$ with $\kappa, \nu \in Q$.

\footnotetext{
${ }^{2}$ The classical locally weakly observability proposed in [13] considers the infinite derivatives, while we add regularity with considering just the first $n-1$ derivatives in the rank condition in this paper.
} 


\subsection{Second basic observability form}

The second basic observability form, i.e. $S^{\prime}(2)$, is inspired by the geometrical results $[15,14]$, and its vector field $g_{q}$ is written as follows:

$$
S^{\prime}(2): \quad g_{q}(z)=\alpha(z)+\gamma_{q}\left(z_{1}\right)
$$

where vector fields $\alpha: V \rightarrow \mathbb{R}^{n}, \gamma_{q}: \mathbb{R} \rightarrow \mathbb{R}^{n}$.

Theorem 2 There exists a diffeomorphism $z=\phi(x)$ which can transform the system $S$ into system $S^{\prime}(2)$, if and only if there exists a family of linearly independent vector fields $\left\{\tau_{1}, \ldots \tau_{n}\right\}$ such that

$$
\begin{aligned}
& \text { 1. } d h \cdot \tau_{1}=1 \\
& \text { 2. } d h \cdot \tau_{i}=0, \forall i \in\{2, \ldots, n\} \\
& \text { 3. }\left[\tau_{i}, \tau_{j}\right]=0, \forall(i, j) \in\{1, \ldots, n\} \times\{1, \ldots, n\} \\
& \text { 4. }\left[\tau_{i}, f_{\Delta_{\kappa, \nu}}\right]=0, \forall i \in\{2, \ldots, n\}, \forall \kappa, \nu \in Q
\end{aligned}
$$

The dynamical systems characterized by the theorem of Krener and Isidori in [15] would fulfill conditions of Theorem 2. It is worth to mention that the class which satisfies conditions of the Theorem 2 is larger than the class which satisfies the theorem of Krener and Isidori, since the fact that we avoid a linear condition for $\alpha$. In Example 1, an academic system which satisfies conditions of Theorem 2 will be given, but it does not satisfy conditions of the theorem in [15].

Remark 1 The previous theorems does not guarantee the uniqueness of vector fields $\tau$. One method to calculate the vector fields $\tau$ is using the following induction method: one can first find the vector field $\tau_{1}$ according to condition $d h \cdot \tau_{1}=1$, then for $\tau_{i}, i \in\{2, \ldots, n\}$, it can be found according to conditions that

- a) $d L_{f_{q}}^{i-1} h \cdot \tau_{i}=1$;

- b) $d L_{f_{q}}^{j} h \cdot \tau_{i}=0, \forall j<i$;

- c) $\left[\tau_{j}, \tau_{i}\right]=0, \forall j<i$.

For the extended observability form presented in the following subsection, the previous method also can be adopted to calculate the vector fields needed in the theorems. Example 1 and 2 are using this method to find such vector fields.

Example 1 Define the dynamics of system $S$ as below

$$
\begin{aligned}
& f_{q}(x)=\left(\begin{array}{c}
\frac{x_{1}+x_{2}}{x_{1}-x_{2}}+\frac{x_{1}}{x_{1}-x_{2}} \mu(q, x) \\
-\frac{x_{1}+x_{2}}{x_{1}-x_{2}}-\frac{x_{2}}{x_{1}-x_{2}} \mu(q, x)
\end{array}\right) \\
& h(x)=x_{1}+x_{2}
\end{aligned}
$$


where $\mu(q, x)=x_{1} x_{2}-\left(x_{1}+x_{2}\right)^{3}+q\left(x_{1}+x_{2}\right)$ and $q \in\{1,2\} . f_{\Delta}(x)=$ $f_{1}(x)-f_{2}(x)=\frac{x_{1}+x_{2}}{x_{1}-x_{2}}\left(x_{1}, x_{2}\right)^{T}$ and $d h=d x_{1}+d x_{2}$. Then $\tau_{1}$ can be calculated $\tau_{1}=\frac{1}{x_{1}-x_{2}}\left(x_{1} \frac{\partial}{\partial x_{1}}-x_{2} \frac{\partial}{\partial x_{2}}\right)$; On the other hand, $d L_{f_{q}} h=\left(x_{2}-3\left(x_{1}+x_{2}\right)^{2}+\right.$ q) $d x_{1}+\left(x_{1}-3\left(x_{1}+x_{2}\right)^{2}+q\right) d x_{2}$ which implies $\tau_{2}=\frac{1}{x_{1}-x_{2}}\left(-\frac{\partial}{\partial x_{1}}+\frac{\partial}{\partial x_{2}}\right)$. Afterwards, it can be easily verified that such vector fields $\tau_{1}, \tau_{2}$ coincide with conditions in Theorem 2.

Meanwhile the diffeomorphism can be given as $z=\phi(x)=\left(x_{1}+x_{2}, x_{1} x_{2}\right)^{T}$, through which the original switched system defined by (6) can be transformed to the form of $S^{\prime}(2)$, written as

$$
\left\{\begin{array}{l}
\dot{z}_{1}=z_{2}-z_{1}^{3}+q z_{1} \\
\dot{z}_{2}=-z_{1} \\
y=z_{1}
\end{array}\right.
$$

\subsection{Extended observability form}

Then we can easily extend the two basic observability forms to a more general form, called the "extended observability form", i.e. $S^{\prime}(3)$, and its vector field $g_{q}$ can be written as follows:

$$
S^{\prime}(3): \quad g_{q}(z)=\left(\begin{array}{c}
g_{q}^{1}(\xi, \eta) \\
g_{q}^{2}(\xi, \eta)
\end{array}\right)
$$

where $\xi \in V_{1} \subset \mathbb{R}^{r}, \eta \in V_{2} \subset \mathbb{R}^{n-r}$ and $z=(\xi, \eta)^{T} ; g_{q}^{1}(\xi, \eta)=A_{r r} \xi+B_{r} \chi_{q}(\xi, \eta)$ with $A_{r r}$ a $r \times r$ superior nilpotent Jordan matrix, and $B_{r}=(0, \ldots, 0,1)^{T} \in \mathbb{R}^{r}$; $g_{q}^{2}(\xi, \eta)=\alpha(\xi, \eta)+\gamma_{q}(\xi)$ with vector fields $\alpha: V_{1} \times V_{2} \rightarrow \mathbb{R}^{n-r}, \gamma_{q}: V_{1} \rightarrow \mathbb{R}^{n-r}$, $\chi_{q}: V_{1} \times V_{2} \rightarrow \mathbb{R}$. The parameter $r$ is the relative degree of the original system when considering $q$ as an input.

Moreover, if $1 \leq r<n, \chi_{q}$ has the following form $\chi_{q}(\xi, \eta)=\alpha^{0}(\xi, \eta)+\gamma_{q}^{0}(\xi)$ with $\alpha^{0}: V_{1} \times V_{2} \rightarrow \mathbb{R}$ and $\gamma_{q}^{0}: V_{1} \rightarrow \mathbb{R}$; but if $r=n, \chi_{q}$ does not have this form and the extended form $S^{\prime}(3)$ degenerates into the first basic form $S^{\prime}(1)$.

Theorem 3 There exists a diffeomorphism $\phi(x)$ which can transform the system $S$ into the system $S^{\prime}(3)$, if and only if $\forall \kappa, \nu \in Q$

1. $L_{f_{\Delta_{\kappa}, \nu}} L_{f_{\kappa}}^{i-1} h(x)=0$, where $\forall i \in\{1, \ldots, r-1\}$;

2. there exist $n-r$ independent vector fields $\left\{\tau_{1}, \ldots, \tau_{n-r}\right\}$, such that
(a) $d L_{f_{\kappa}}^{i-1} h \cdot \tau_{j}=0, \quad i \in\{1, \ldots, r\}, j \in\{1, \ldots, n-r\}$;
(b) $\left[\tau_{i}, \tau_{j}\right]=0, \quad i, j \in\{1, \ldots, n-r\}$;
(c) $\left[\tau_{i}, f_{\Delta_{\kappa, \nu}}\right]=0, \quad i \in\{1, \ldots, n-r\}$.

where $0<r \leq n$. 
Example 2 The dynamics of system $S$ is now given by:

$$
\begin{aligned}
& f_{q}(x)=\left(\begin{array}{l}
x_{2}+x_{3}-\mu(q, x) \\
\frac{x_{2}+x_{3}}{x_{2}-x_{3}}+\frac{x_{2}}{x_{2}-x_{3}} \mu(q, x) \\
-\frac{x_{2}+x_{3}}{x_{2}-x_{3}}-\frac{x_{3}}{x_{2}-x_{3}} \mu(q, x)
\end{array}\right) \\
& h(x)=x_{1}+x_{2}+x_{3}
\end{aligned}
$$

where $\mu(q, x)=x_{2} x_{3}-\left(x_{2}+x_{3}\right)^{3}+q\left(x_{2}+x_{3}\right), q \in\{1,2\}$ and $f_{\Delta}(x)=f_{1}(x)-$ $f_{2}(x)=\frac{x_{2}+x_{3}}{x_{2}-x_{3}}\left(-\frac{\partial}{\partial x_{1}}+x_{2} \frac{\partial}{\partial x_{2}}-x_{3} \frac{\partial}{\partial x_{3}}\right)$. Then condition 1) can be easily verified with $r=2$. Then, $d L_{f_{q}}^{r} h=\left(x_{3}-3\left(x_{2}+x_{3}\right)^{2}+q\right) d x_{2},\left(x_{2}-3\left(x_{2}+x_{3}\right)^{2}+q\right) d x_{3}$, and according to Remark 1 , the only restriction for $\tau_{1}$ becomes to $d L_{f_{q}}^{r} h \cdot \tau_{1}=1$, so we can find $\tau_{1}=-\frac{1}{x_{2}-x_{3}}\left(\frac{\partial}{\partial x_{2}}-\frac{\partial}{\partial x_{3}}\right)$, which verifies conditions $\left.2 a\right)$, 2b), and 2c).

Consequently, there exists a diffeomorphism $\phi$ defined as $z=\phi(x)=\left(x_{1}+x_{2}+x_{3}, x_{2}+x_{3}, x_{2} x_{3}\right)^{T}$ and it can transform system $S$ to $S^{\prime}(3)$ written as below

$$
\left\{\begin{array}{l}
\dot{z}_{1}=z_{2} \\
\dot{z}_{2}=z_{3}-z_{2}^{3}+q z_{2} \\
\dot{z}_{3}=-z_{2} \\
y=z_{1}
\end{array}\right.
$$

\subsection{Proofs for theorems}

\section{Proof 1 (1. Proof of Theorem 3) Sufficiency:}

Condition 1) implies that the relative degree is $r$. According to the theory of nonlinear feedback for SISO system in [14], the following function $\phi_{1}: U \rightarrow V_{1}$

$$
\phi_{1}=\left(h(x), L_{f_{\kappa}} h(x), \ldots, L_{f_{\kappa}}^{r-1} h(x)\right)^{T}
$$

is of rank $r$ such that the first $r$ states in the new coordinate frame $\xi=\phi_{1}(x) \in$ $V_{1}$ are with the form of $\left(g_{q}\right)_{1}(\xi)$.

Condition 2b) implies that there exists a smooth function $\phi_{2}: U \rightarrow V_{2}$

$$
\phi_{2}=\left(\eta_{1}(x), \eta_{2}(x), \ldots, \eta_{n-r}(x)\right)^{T}
$$

such that

$$
\begin{aligned}
& d \eta_{i} \cdot \tau_{i}=1, \forall i \in\{1, \ldots, n-r\} \\
& d \eta_{i} \cdot \tau_{j}=0, \forall i, j \in\{1, \ldots, n-r\}, \text { and } i \neq j .
\end{aligned}
$$

Moreover, condition 2a) implies that the one form $d \phi_{1}$ is independent to the one form $d \phi_{2}$, and one can set that $\phi=\left(\phi_{1}, \phi_{2}\right)^{T}$ is a diffeomorphism defined from $U \rightarrow V_{1} \times V_{2}$ such that

$$
\phi_{*}\left(\tau_{i}\right):=d \phi \cdot \tau_{i}=\left(\begin{array}{c}
d \phi_{1} \\
d \phi_{2}
\end{array}\right) \cdot \tau_{i}=\frac{\partial}{\partial \eta_{i}}
$$


According to the transformation of Lie bracket by mean of addiffeomorphism, we have

$$
\frac{\partial}{\partial \eta_{i}}\left(\phi_{*}\left(f_{\Delta_{\kappa, \nu}}\right)\right)=\left[\phi_{*}\left(\tau_{i}\right), \phi_{*}\left(f_{\Delta_{\kappa, \nu}}\right)\right]=0 \quad \forall i \in\{1, \ldots, n-r\}
$$

where $\phi_{*}$ represents the Jacobian Matrix.

This result means that in the new coordinate frame, we obtain

$$
\phi_{*}\left(f_{\Delta_{\kappa, \nu}}\right)=\sum_{i=1}^{n-r} \varphi_{i}(\xi) \frac{\partial}{\partial \eta_{i}}
$$

which means that $\dot{\eta}$ is in the form $g_{q}^{2}(\xi, \eta)$, and also $\chi_{q}$ has the form $\chi_{q}(\xi, \eta)=$ $\alpha^{0}(\xi, \eta)+\gamma_{q}^{0}(\xi)$.

Necessity: If $r=n$, the form $S^{\prime}(3)$ will degenerate into the first basic form $S^{\prime}(1)$, and suppose there exists a diffeomorphism $z=\phi(x)$ which can transforms the system $S$ into the form $S^{\prime}(1)$.

One can easily conclude that the form $S^{\prime}(1)$ satisfies Assumption 1 and (4).

$$
L_{\tilde{f}_{\Delta_{\kappa}, \nu}} L_{\tilde{f}_{\kappa}}^{i-1} \tilde{h}=0
$$

where $\tilde{f}_{q}=A_{n n} z+B_{n} \chi_{q}(z), \tilde{f}_{\Delta_{\kappa, \nu}}=\tilde{f}_{\kappa}-\tilde{f}_{\nu}$, and $\tilde{h}=z_{1}$.

Obviously, it has

$$
\left.f_{q}\right|_{x}=\left.\left(\frac{\partial \phi}{\partial x}\right)^{-1} \tilde{f}_{q}\right|_{z=\phi(x)}
$$

where $\left.f_{q}\right|_{x}$ denotes function $f_{q}$ in $x$ coordinate system. Consequently,

$$
L_{f_{\Delta_{\kappa}, \nu}} L_{f_{\kappa}}^{i-1} h=L_{\tilde{f}_{\Delta_{\kappa}, \nu}} L_{\tilde{f}_{\kappa}}^{i-1} \tilde{h}=0
$$

If $1 \leq r<n$ then suppose that there exists a diffeomorphism $\phi(x)$ which transforms $S$ into $S^{\prime}(3)$. Let's write $\xi=\phi_{1}(x) \in V_{1}$ and $\eta=\phi_{2}(x) \in V_{2}$, where $\phi=\left(\begin{array}{l}\phi_{1} \\ \phi_{2}\end{array}\right)$. From the first part of dynamics of $S^{\prime}(3)$, it can be concluded that the relative degree is $r$ which implies condition 1). Then denote a set of vector fields $\pi:=\left\{\pi_{1}, \ldots, \pi_{n}\right\}$ the columns of $\left(\frac{\partial \phi}{\partial x}\right)^{-1}$ and define another set of vector fields $\left\{\tau_{1}, \ldots, \tau_{n-r}\right\}$, where $\tau_{i}=\pi_{r+i}$. Obviously, conditions 2a) and 2b) are satisfied with vector fields $\tau_{i}$ such that $\left[\tau_{i}, \pi_{j}\right]=0, \forall i, j$.

Consider the $(\xi, \eta)$ coordinate frame, there exists a set of functions $\varphi_{i}^{\kappa, \nu}(\xi) \in$ $\mathbb{R}$, such that

$$
\left.f_{\Delta_{\kappa, \nu}}\right|_{(\xi, \eta)}=\sum_{i=1}^{n-r} \varphi_{i}^{\kappa, \nu}(\xi) \frac{\partial}{\partial \eta_{i}}+\gamma_{\Delta_{\kappa, \nu}}^{0}(\xi) \frac{\partial}{\partial \xi_{r}}
$$

where $\gamma_{\Delta_{\kappa, \nu}}^{0}(\xi)=\gamma_{\kappa}^{0}(\xi)-\gamma_{\nu}^{0}(\xi)$ with $\kappa, \nu \in Q$.

Then, rewrite equation (11) in original $x$ coordinate frame

$$
\left.f_{\Delta_{\kappa, \nu}}\right|_{x}=\sum_{i=1}^{n-r} \varphi_{i}^{\kappa, \nu}(\xi) \tau_{i}+\left.\gamma_{\Delta_{\kappa, \nu}}^{0}(\xi) \pi_{r}\right|_{\xi=\phi_{1}(x)}
$$

which implies condition 2c). 
Proof 2 (2. Proof of Theorem 1 and Theorem 2) When $r=n$, it is just the case of form $S^{\prime}(1)$ and Theorem 1 can be easily proved by setting $r=n$ in Theorem 3.

While if $r=1$, condition 1) is not valid any more and it degenerates into the form $S^{\prime}(2)$, meanwhile, if setting $z_{1}=h(x)$, associating with condition 2a) in Theorem 3, one will obtain the same conditions in Theorem 2. Consequently, Theorem 2 can also be proved by setting $r=1$ and providing just one additional condition $z_{1}=h(x)$.

\section{Observability analysis}

Before analyzing the observability of the system $S$, it is important to emphasize that instead of the full knowledge of the discrete state $q$, only the partial knowledge - the value of the filtered discrete state (often a low pass filter) can be obtained. This is due to the fact that the sensor normally has relative low speed comparing to the switching frequency considered in this paper, since it's Zeno (or high switching frequency). In practice, a low pass filter $\mathcal{F}$ is often adopted before the sensor, and the passed signal, denote $q_{\mathcal{F}}$, can be approximated as the time average of discrete state $q$, while the oscillations written as $q-q_{\mathcal{F}}$ cannot pass the filter $\mathcal{F}$, see Fig. 1.

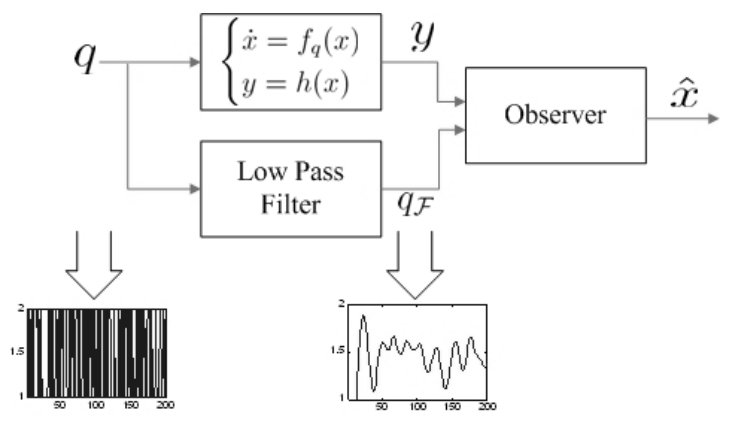

Figure 1: Diagram of switched system and its observer; The discrete state $q$ (left plot) and its filtered value $q_{\mathcal{F}}$ (right plot).

What if the exact switching signal is not available but its filtered value can be captured? Before answer this question, let us recall some conceptions on integral.

\subsection{Recall of Henstock-Kurzweil Integral and its implica- tions for Zeno}

Henstock-Kurzweil integral (H-K integral) [4, 12], also known as the gauge integral, is particularly a generalization of the well-known Riemann integral. 
Definition 1 (Riemann (respectively H-K) integrable) A number I is called the Riemann integral (respectively the $H$-K integral) of $f:[a, b] \rightarrow \mathbb{R}$ if for each constant $\varepsilon>0$, there exists a constant $\delta$ (respectively a function $\delta:[a, b] \rightarrow \mathbb{R}_{+}$), such that whenever $n$ is a positive integer and $t_{0}, t_{1}, \ldots, t_{n}$ and $s_{1}, s_{2}, \ldots, s_{n}$ are some numbers satisfying

$$
a=t_{0} \leq s_{1} \leq t_{1} \leq s_{2} \leq t_{2} \leq \ldots \leq s_{n} \leq t_{n}=b
$$

and $t_{i}-t_{i-1}<\delta$ (respectively $\left.t_{i}-t_{i-1}<\delta\left(s_{i}\right)\right)$ for all $i$, then $\left|I-\sum_{i=1}^{n} f\left(s_{i}\right)\left(t_{i}-t_{i-1}\right)\right|<$ $\varepsilon$.

Apparently, the only difference between Riemann integral and H-K integral is that the gauge $\delta$ in $\mathrm{H}-\mathrm{K}$ integral is a function of $s_{i}$ instead of constant value in Riemann integral. Taking a more general form of the gauge $\delta$ (instead of $\delta$ constant) yields a rich class of possible integrands and permits to obtain that

\section{Riemann integrable functions $\subset$ Lebesgue integrable functions $\subset H$-K integrable functions}

To make the problem much simpler, without loss of generality, we assume that system $S$ is affine with respect to the discrete state $q$, hence $\gamma_{q}(y)=\gamma(y) q$. Meanwhile define

$$
\dot{p} \triangleq \gamma(y)\left(q-q_{\mathcal{F}}\right)
$$

Then, according to the characteristics of Zeno phenomenon, such as the Chattering Zeno (i.e. after some time the dwell time is exactly equal to zero) or the Genuinely Zeno (i.e. the dwell time is never equal to zero) [1], we can make the following assumption:

Assumption 2 The switching terms $\gamma(y) q$ and $\gamma(y) q_{\mathcal{F}}$ are both $H$-K integrable over $\left[t_{0}, t_{1}\right] \subset \mathbb{R}_{+}$, where $\left[t_{0}, t_{1}\right]$ is the interval with Zeno phenomenon or high switching frequency.

Assumption 2 implies that $\gamma(y)\left(q-q_{\mathcal{F}}\right)$ is H-K integrable, hence, $\forall \varepsilon>0$, it has

$$
\|p\|=\sup _{t \in\left[t_{0}, t_{1}\right]}\left|\int_{t_{0}}^{t} \gamma(y)\left(q-q_{\mathcal{F}}\right) d \tau\right|<\varepsilon
$$

where $t_{1}>t_{0} \geq 0, t \in\left[t_{0}, t_{1}\right]$ and the integration is in the sense of H-K.

In [5], the authors have introduced Caratheodory solutions and Fillipov solutions for discontinuous systems. Analogically, the solution for the system $S$ can be defined in the same way but in the sense of H-K integral, for example, we can define its Caratheodory solutions as

$$
x(t)=x\left(t_{0}\right)+\int_{t_{0}}^{t} f_{q}(x(s)) d s, \quad t>t_{0}
$$

where the integral is H-K integral for almost everywhere. Since the fact that H-K integral is much more general than Lebesgue (or Riemann) integral, properties of function for Lebesgue integral are also satisfied for H-K integral. And this is the same for Fillipov solutions. 
Remark 2 From Assumption 2, one can also obtain that $\gamma(y)$ is $H$-K integrable. But it is not sufficient only assuming that $\gamma(y)$ is $H-K$ integrable. To highlight this point, one example is given below.

Example 3 Define $\gamma:\left[0, b\left[\rightarrow \mathbb{R}\right.\right.$, with $\gamma(y)=\frac{1}{y} \sin \frac{1}{y^{2}}$ for $y \neq 0$ and $\gamma(0)=0$. Hence $\gamma$ is $H$-K integrable [12]. Now considering the following dynamic:

$$
\dot{p}=\gamma(y)\left(q-q_{\mathcal{F}}\right)=\frac{1}{y} \sin \frac{1}{y^{2}}\left(q-q_{\mathcal{F}}\right)
$$

which has a Caratheodory solution in the $H$-K meaning for sufficiently smooth $\left(q-q_{\mathcal{F}}\right)$, but does not have Caratheodory solution if the considered integral are restricted to Lebesgue. Nevertheless, it is important to mention that for a particular $\left(q-q_{\mathcal{F}}\right)$, as for example $q-q_{\mathcal{F}}=\operatorname{sign}\left(\sin \frac{1}{y^{2}}\right)$ equation (14) has also no Caratheodory solution in the $H-K$ meaning.

\subsection{Observability analysis}

As discussed above, H-K integral includes Riemann and Lebesgue integral. Therefor, we adopt this integral definition here in order to treat the more general case, even if Lebesgue integral is enough at most of time.

Then define the observability as follows:

Definition 2 Constructively Regularly Weakly Locally Observability: Switched system with Zeno phenomenon or high switching frequency is said to be Constructively Regularly Weakly Locally Observable (CRWLO), if there exists at least one observer for the considered system such that the error between the observation and the original state is locally asymptotically stable.

\subsubsection{Observability for the first basic form}

Theorem 4 Under Assumption 1, the form $S^{\prime}(1)$ with Zeno phenomenon or high switching frequency, is CRWLO.

Theorem 4 is evident, since the observability of the continuous states $z$ are independent to the discrete state $q$. Moreover, RWLO can be guaranteed even without any knowledge on $q$ for this form.

\subsubsection{Observability for the second basic form}

In order to analyze the observability for the second basic form, we need two more assumptions:

Assumption 3 The pair $(y, \alpha(z))$ of form $S^{\prime}(2)$ is $C R W L O$.

Assumption 1 and Assumption 3 imply that there exists an observer $\hat{z}$ for the pair $(y, \alpha(z))$, given by

$$
\begin{aligned}
\dot{\hat{z}} & =\beta(\hat{z}, y, \hat{y}) \\
\hat{y} & =\hat{z}_{1}
\end{aligned}
$$


and the continuous state observation error $e=z-\hat{z}$ is exponentially stable, so there exists a Lyapunov function $V(e)$ such that

$$
\dot{V}(e)=\frac{\partial V}{\partial e}(\alpha(z)-\beta(\hat{z}, y, \hat{y}))<-K V(e)
$$

where $K>0$. Then, with the same Lyapunov function $V$ and the observer $\hat{z}$ for the form $S^{\prime}(2)$ designed as follows

$$
\dot{\hat{z}}=\beta(\hat{z}, y, \hat{y})+\gamma(y) q_{\mathcal{F}}
$$

Theorem 5 Under Assumptions 1, 2, 3 and with the knowledge of $q_{\mathcal{F}}$, the form $S^{\prime}(2)$ with Zeno phenomenon or high switching frequency, is CRWLO.

Proof 3 According to (18), the corresponding observation error e of continuous state will change to

$$
\dot{e}=\alpha(z)-\beta(\hat{z}, y, \hat{y})+\gamma(y)\left(q-q_{\mathcal{F}}\right)
$$

Let $\epsilon=e-p$, where $p$ is defined in (12), then dynamic of $\epsilon$ can be written

$$
\dot{\epsilon}=\alpha(z)-\beta(\hat{z}, y, \hat{y})
$$

Adopt the same Lyapunov function $V$ of observer (15)-(16) on $\epsilon$, and compute its derivation

$$
\dot{V}=\frac{\partial V}{\partial \epsilon}(\alpha(z)-\beta(\hat{z}, y, \hat{y}))
$$

Applying Taylor expansion on vector $\frac{\partial V}{\partial \epsilon}$ around $-p$, one can easily obtain

$$
\begin{aligned}
\dot{V}= & \left.\frac{\partial V}{\partial e}\right|_{e}(\alpha(z)-\beta(\hat{z}, y, \hat{y}))- \\
& \left.\frac{\partial^{2} V}{\partial e^{2}}\right|_{e}(O(p) \otimes(\alpha(z)-\beta(\hat{z}, y, \hat{y})))+O\left(\|p\|^{2}\right)
\end{aligned}
$$

where $\lim _{\|p\| \rightarrow 0} O(\|p\|)=0$. Then thanks to (13) and (17), it has

$$
\begin{aligned}
\dot{V}< & -K V(e)+\left|\frac{\partial^{2} V}{\partial e^{2}}\right|_{e}(O(p) \otimes(\alpha(z)-\beta(\hat{z}, y, \hat{y}))) \mid+ \\
& \left|O\left(\|p\|^{2}\right)\right| \\
< & -K V(e)+|O(\varepsilon)|
\end{aligned}
$$

Consequently, it is possible to set $\varepsilon \ll V_{d}$ which guarantees that there exists a small $M>0$ such that $|O(\varepsilon)|<M V_{d}$. Then $\forall V_{d}>0$, and $\forall e \in\left\{e: V(e) \geq V_{d}\right\}$, it has

$$
\dot{V}<-K^{\prime} V(e)
$$

where $K^{\prime}=K-M>0$. 


\subsubsection{Observability for the extended form}

The extended form is just the composition of the two basic forms, for which the observability have been discussed in the previous subsections. Consequently, one can easily conclude the following proposition.

Proposition 6 Under Assumptions 1, 2, 3 and with the knowledge of $q_{\mathcal{F}}$, the form $S^{\prime}(3)$ with Zeno phenomenon or high switching frequency, is CRWLO.

\subsection{Observability for system $S$}

The following Corollary can be directly concluded from Theorem 1, 2, 3, 4, 5 and Proposition 6.

Corollary 7 Under Assumptions 1, 2, 3 and with the knowledge of $q_{\mathcal{F}}$, system $S$ with Zeno phenomenon or high switching frequency is CRWLO, if conditions in Theorem 1 (or Theorem 2, 3) are satisfied.

\section{Simulations}

The objective of this section is to highlight the efficiencies of the observability for proposed forms with Zeno phenomenon or high switching frequency. Without loss of generality, we just use the same example expressed in Example 1 and 2, while we build a new example for the first basic form. To simulate the Zeno phenomenon, the discrete state $q$ is driven by a function defined as $\sigma(t)=\frac{3+\operatorname{sign}(w(t))}{2}$ with $w(t)$ the Gaussian random process. Afterwards, to simulate the filtered discrete signal $q_{\mathcal{F}}$, a low pass filter with cutoff frequency $f_{c}=100 \mathrm{~Hz}$ is used, where we must guarantee that $f_{c}$ is bigger than the bandwidth of system. At the end, to built the observer, ALIEN method (an numerical algebraic differential method) [18], high gain method [3] and sliding mode method [10] are respectively adopted. All ODEs in simulations are implemented with solver "db45".

Simulation 1 (The first basic observability form) To verify the observability of form $S^{\prime}(1)$, we design a simple system as

$$
\left\{\begin{array}{l}
\dot{z}_{1}=z_{2} \\
\dot{z}_{2}=-2 z_{1}+z_{1} q \\
y=z_{1}
\end{array}\right.
$$

Easily, one can apply an observer based on the second order sliding mode method to estimate $z_{2}$. And the simulation results are shown in Fig. 2(A).

Simulation 2 (The second basic observability form) Using high gain method to build an observer for system (7), we can easily write the observer as below

$$
\dot{\hat{z}}=\alpha(\hat{z})+\gamma_{q_{\mathcal{F}}}(y)-K\left(\hat{z}_{1}-y\right)
$$

where $K=(100,100)^{T}$. The observation for $z_{2}$ is shown in Fig. 2(B). 


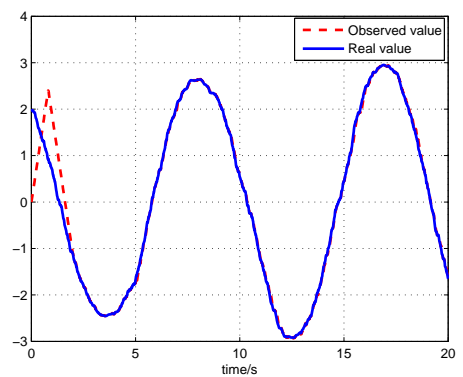

$A$

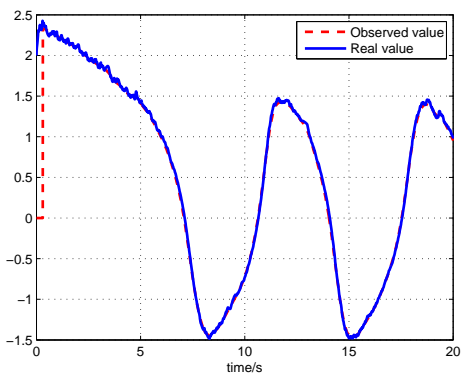

C

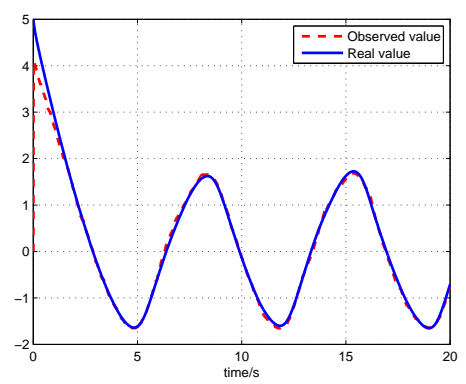

$B$

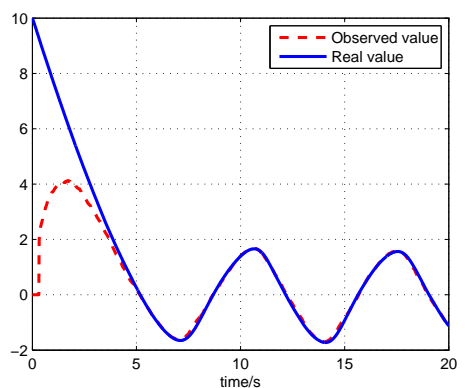

$D$

Figure 2: A: $z_{2}$ of system (19) and its observation using sliding mode with $\hat{z}_{2}(0)=0 ; \mathrm{B}: \hat{z}_{2}$ of system (7) and its observation using high gain method with $\hat{z}_{2}(0)=0$; C: $\hat{z}_{2}$ of system (10) and its observation using ALIEN method with $\hat{z}_{2}(0)=0$; D: $\hat{z}_{3}$ of system (10) and its observation using high gain method with $\hat{z}_{3}(0)=0$.

Simulation 3 (The extended observability form) Considering system (10), we use ALIEN method to observe $z_{2}$ and high gain method to observe $z_{3}$ with $K=(100,100)^{T}$. The results ${ }^{3}$ are shown in Fig. 2(C,D).

\section{Conclusion}

This paper mainly discusses the observability, defined as Constructive Regularly Weakly Locally Observability (CRWLO), for a class of switched system with Zeno phenomenon or high switching frequency. Particularly, three observability forms are proposed, and sufficient and necessary conditions for the existence of diffeomorphism that can transform systems into each form are presented. On the other hand, CRWLO for each forms are proved under some reasonable

\footnotetext{
${ }^{3}$ The time delay for observation using ALIEN method has been compensated in all simulations, and the technology for compensation is presented in [18].
} 
assumptions. Moreover, open problems such as the case of multi-output or switching with state jumps also can be investigated in the same method proposed in this paper.

\section{References}

[1] A. D. Ames, H. Zheng, R. D. Gregg, and S. Sastry. Is there life after zeno? taking executions past the breaking (zeno) point. In Proc. American Control Conference, page 6pp., 14-16 June 2006.

[2] J. P. Aubin and A. Cellina. Differential inclusions, set-valued maps and viability theory. Grundl. der Math. Wiss., 264, 1984.

[3] G. Besançon, Q. Zhang, and H. Hammouri. High-gain observer based state and parameter estimation in nonlinear systems. In 6th IFAC symposium on Nonlinear Control Systems, 2004.

[4] M. Cichon, I. Kubiaczyk, and A. Sikorska. The henstock-kurweil-pettis integral and existence theorems for the cauchy problem. Czeckoslovak Mathematical Journal, 54:279-289, 2004.

[5] Jorge Cortes. Discontinuous dynamical systems. Control Systems Magazine, 28(3):36-73, 2008.

[6] B. Drazenovic. The invariance condition in variable structure systems. Automatica, 5(3):287-295, 1969.

[7] S. V. Emelyanov and S. K. Korovin. Applying the principle of control by deviation to extend the set of possible feedback types. Soviet Physics, Doklady, 26(6):562-564, 1981.

[8] A. F. Filippov. Classical solutions of differential equations with multivalued right-hand sides. SIAM J. Control, 5:609-621, 1967.

[9] A. F. Filippov. Differential Equations with Discontinuous Righthand sides. Kluwer Academic Publishers, 1988.

[10] T. Floquet and J. P. Barbot. Super twisting algorithm-based step-bystep sliding mode observers for nonlinear systems with unknown inputs. International Journal of Systems Science, 38(10):803-815, 2007.

[11] R. Goebel, R. G. Sanfelice, and Andrew R. Teel. Hybrid dynamical systems. IEEE Contr. Syst. Mag., 29(2):28-93, March 2009.

[12] R. Henstock. The General Theory of Integration. Oxford Mathematical Monographs, Clarendon Press, 1991.

[13] R. Hermann and A. J. Krener. Nonlinear controllability and observability. IEEE Transactions on Automatic Control, 22:728-740, 1977. 
[14] A. Isidori. Nonlinear Control Systems. Communication and Control Engineering Series, Third Edition, Springer-Verlag, 1995.

[15] A. J. Krener and A. Isidori. Linearization by output injection and nonlinear observer. Sys. \& Control Letters, 3:47-52, 1983.

[16] A. Levant. Sliding order and sliding accuracy in sliding mode control. Int. J. of Control, 34(6):1253-1253, 1993.

[17] Daniel Liberzon. Switching in Systems and Control. Systems \& Control: Foundations \& Applications. Birkhäuser, 2003.

[18] M. Mboup, C. Join, and M. Fliess. A revised look at numerical differentiation with an application to nonlinear feedback control. In The 15th Mediterrean Conference on Control and Automation (MED'2007), 2007.

[19] W. Perruquetti and J. P. Barbot. Sliding Mode Control in Engineering. Ed. Marcel Dekker, 2002.

[20] V. Utkin. Sliding Modes in Optimization and Control. Springer-Verlag, 1992.

[21] L. Yu, J. P. Barbot, D. Boutat, and D. Benmerzouk. Observability normal forms for a class of switched systems wih zeno phenomena. In IEEE ACC, 2009.

[22] J. Zhang, K. H. Johansson, J. Lygeros, and S. Sastry. Zeno hybrid systems. International Journal of Robust and Nonlinear Control, 11:435-451, 2001. 